\title{
DETERMINATION OF SURFACE LOCATION BETWEEN TWO SUB-DOMAINS USING THE TEMPERATURE DISTRIBUTION ON THE EXTERNAL SURFACE
}

\author{
Katarzyna Freus ${ }^{1}$, Sebastian Freus ${ }^{2}$ \\ ${ }^{I}$ Institute of Mathematics, Czestochowa University of Technology \\ ${ }^{2}$ Institute of Computer and Information Science, Czestochowa University of Technology \\ Czestochowa, Poland \\ katarzyna.freus@im.pcz.pl,sebastian.freus@icis.pcz.pl
}

\begin{abstract}
In the paper two sub-domains which are in thermal contact are considered. The temperature field in these domains is described by the system of two Laplace equations supplemented by the boundary conditions. The position of surface between sub-domains is unknown. Additional information necessary to solve the identification problem results from a knowledge of external surface temperature distribution. The direct problem is solved using the boundary element method. To solve the inverse problem formulated the gradient method is applied. In the final part of the paper the results of computations are shown. The algorithm proposed here can be used, among others, in the medical practice (e.g. in burns therapy).
\end{abstract}

\section{Direct problem}

The temperature field in the domains is described by equations

$$
(x, y) \in \Omega_{e}: \quad \lambda_{e} \frac{\partial^{2} T_{e}(x, y)}{\partial x^{2}}+\lambda_{e} \frac{\partial^{2} T_{e}(x, y)}{\partial y^{2}}=0, \quad e=1,2
$$

where $\lambda_{e}[\mathrm{~W} /(\mathrm{mK})]$ is the thermal conductivity of sub-domain $\Omega_{e}, T_{e}, x, y$ denote the temperature and the geometrical co-ordinates, respectively.

On the external surface (c.f. Fig. 1) the Robin condition is known

$$
(x, y) \in \Gamma_{e x}: \quad-\lambda_{1} \frac{\partial T_{1}(x, y)}{\partial n}=\alpha\left[T_{1}(x, y)-T_{a}\right]
$$

where $T_{a}$ is the ambient temperature, $\alpha$ is the heat transfer coefficient, $\partial T_{1} / \partial n$ denotes the normal derivative. On the internal surface $\Gamma_{i n}$ (c.f. Fig. 1) the Dirichlet condition can be taken into account

$$
(x, y) \in \Gamma_{\text {in }}: \quad T_{2}(x, y)=T_{b}
$$


On the surface between sub-domains the ideal contact is assumed

$$
(x, y) \in \Gamma_{c}:\left\{\begin{aligned}
-\lambda_{1} \frac{\partial T_{1}(x, y)}{\partial n} & =\lambda_{2} \frac{\partial T_{2}(x, y)}{\partial n} \\
T_{1}(x, y) & =T_{2}(x, y)
\end{aligned}\right.
$$

On the remaining boundaries the no-flux condition is accepted.

The shape of internal surface $\Gamma_{c}$ is defined by the NURBS curve

$$
\Gamma_{c}(t)=\frac{(1-t)^{2} w_{0} \mathbf{P}_{0}+2 t(1-t) w_{1} \mathbf{P}_{1}+t^{2} w_{2} \mathbf{P}_{2}}{(1-t)^{2} w_{0}+2 t(1-t) w_{1}+t^{2} w_{2}}, \quad 0 \leq t \leq 1
$$

where $\mathbf{P}_{0}, \mathbf{P}_{1}, \mathbf{P}_{2}$ are the control points, $w_{1}, w_{2}, w_{3}$ are the weights [1].

In Figure 1 the position of internal surface corresponds to the control points $\mathbf{P}_{0}=(0,0.016), \mathbf{P}_{1}=(0.01,0.009), \mathbf{P}_{2}=(0.04,0.016)$, the weights $w_{1}=w_{2}=w_{3}=1$.

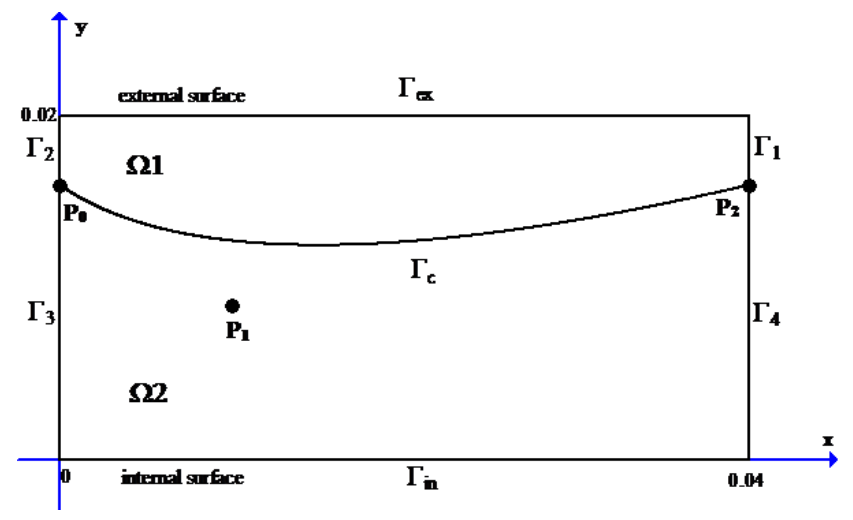

Fig. 1. Domain considered

The direct problem above formulated is solved by means of the BEM.

\section{Boundary element method}

At first the homogeneous domain $\Omega$ is considered. In this case the boundary element method for the Laplace equation leads to the system of equations which for linear boundary elements is the following $(i=1,2, \ldots, R)[2,3]$

$$
\sum_{r=1}^{R} G_{i r} q_{r}=\sum_{r=1}^{R} \hat{H}_{i r} T_{r}
$$

where for the single node $r$ being the end of the boundary element $\Gamma_{j}$ and being the beginning of the boundary element $\Gamma_{j+1}$ one has 


$$
G_{i r}=G_{i j}^{k}+G_{i j+1}^{p}, \quad \hat{H}_{i r}=\hat{H}_{i j}^{k}+\hat{H}_{i j+1}^{p}
$$

while for double node $r, r+1$

$$
\begin{aligned}
& G_{i r}=G_{i j}^{k}, \quad G_{i r+1}=G_{i j+1}^{p} \\
& \hat{H}_{i r}=\hat{H}_{i j}^{k}, \quad \hat{H}_{i r+1}=\hat{H}_{i j+1}^{p}
\end{aligned}
$$

where

$$
\begin{aligned}
G_{i j}^{p} & =\frac{l_{j}}{4 \pi \lambda} \int_{-1}^{1} N_{p} \ln \frac{1}{r_{i j}} \mathrm{~d} \theta \\
G_{i j}^{k} & =\frac{l_{j}}{4 \pi \lambda} \int_{-1}^{1} N_{k} \ln \frac{1}{r_{i j}} \mathrm{~d} \theta
\end{aligned}
$$

and

$$
\begin{aligned}
\hat{H}_{i j}^{p} & =\frac{1}{4 \pi} \int_{-1}^{1} N_{p} \frac{r_{x}^{j} l_{y}^{j}-r_{y}^{j} l_{x}^{j}}{r_{i j}^{2}} \mathrm{~d} \theta \\
\hat{H}_{i j}^{k} & =\frac{1}{4 \pi} \int_{-1}^{1} N_{k} \frac{r_{x}^{j} l_{y}^{j}-r_{y}^{j} l_{x}^{j}}{r_{i j}^{2}} \mathrm{~d} \theta
\end{aligned}
$$

where

$$
\begin{gathered}
r_{i j}=\sqrt{\left(N_{p} x_{j}^{p}+N_{k} x_{j}^{k}-\xi_{i}\right)^{2}+\left(N_{p} y_{j}^{p}+N_{k} y_{j}^{k}-\eta_{i}\right)^{2}}=\sqrt{\left(r_{x}^{j}\right)^{2}+\left(r_{y}^{j}\right)^{2}} \\
l_{j}=\sqrt{\left(x_{j}^{k}-x_{j}^{p}\right)^{2}+\left(y_{j}^{k}-y_{j}^{p}\right)^{2}}=\sqrt{\left(l_{x}^{j}\right)^{2}+\left(l_{y}^{j}\right)^{2}}
\end{gathered}
$$

In formula (13) $N_{p}=(1-\theta) / 2, N_{k}=(1+\theta) / 2, \theta \in[-1,1]$ are the shape functions, $\left(\xi_{i}, \eta_{i}\right)$ is the observation point, $\left(x_{j}^{p}, y_{j}^{p}\right),\left(x_{j}^{k}, y_{j}^{k}\right)$ are the co-ordinates of beginning and end of element $\Gamma_{j}$.

It should be pointed out that if $\left(\xi_{i}, \eta_{i}\right)$ is the beginning of boundary element $\Gamma_{j}$, this means $\left(\xi_{i}, \eta_{i}\right)=\left(x_{j}^{p}, y_{j}^{p}\right)$ then

$$
G_{i j}^{p}=\frac{l_{j}\left(3-2 \ln l_{j}\right)}{8 \pi \lambda}, \quad G_{i j}^{k}=\frac{l_{j}\left(1-2 \ln l_{j}\right)}{8 \pi \lambda}, \quad \hat{H}_{i j}^{p}=\hat{H}_{i j}^{k}=0
$$

while if $\left(\xi_{i}, \eta_{i}\right)$ is the end of boundary element $\Gamma_{j}:\left(\xi_{i}, \eta_{i}\right)=\left(x_{j}^{k}, y_{j}^{k}\right)$ then

$$
G_{i j}^{p}=\frac{l_{j}\left(1-2 \ln l_{j}\right)}{8 \pi \lambda}, \quad G_{i j}^{k}=\frac{l_{j}\left(3-2 \ln l_{j}\right)}{8 \pi \lambda}, \quad \hat{H}_{i j}^{p}=\hat{H}_{i j}^{k}=0
$$


The system of equations (6) can be written in the form

$$
\mathbf{G q}=\mathbf{H T}
$$

where

$$
H_{i r}= \begin{cases}\hat{H}_{i r} & i \neq r \\ -\sum_{\substack{r=1 \\ r \neq i}}^{R} \hat{H}_{i r} & i=r\end{cases}
$$

In the case of non-homogeneous domain $\Omega=\Omega_{1} \cup \Omega_{2}$ two systems of equations for each sub-domain, should be taken into account separately.

So, the following denotations are introduced (c.f. Fig. 1):

- $\mathbf{T}_{1}^{1}, \quad \mathbf{T}_{1}^{2}, \quad \mathbf{T}_{1}^{\text {ex }}, \quad \mathbf{q}_{1}^{1}, \quad \mathbf{q}_{1}^{2}, \quad \mathbf{q}_{1}^{e x}$ are the vectors of functions $T$ and $q$ at the boundary $\Gamma_{1} \cup \Gamma_{2} \cup \Gamma_{e x}$ of domain $\Omega_{1}$,

- $\mathbf{T}_{c 1}, \quad \mathbf{T}_{c 2}, \quad \mathbf{q}_{c 1}, \quad \mathbf{q}_{c 2}$ are the vectors of functions $T$ and $q$ on the contact surface $\Gamma_{c}$ between sub-domains $\Omega_{1}$ and $\Omega_{2}$,

- $\mathbf{T}_{2}^{3}, \quad \mathbf{T}_{2}^{4}, \quad \mathbf{T}_{2}^{\text {in }}, \mathbf{q}_{2}^{3}, \quad \mathbf{q}_{2}^{4}, \quad \mathbf{q}_{2}^{\text {in }}$ are the vectors of functions $T$ and $q$ at the boundary $\Gamma_{3} \cup \Gamma_{4} \cup \Gamma_{\text {in }}$ of domain $\Omega_{2}$.

Using above notations, one obtains the following systems of equations

- for sub-domain $\Omega_{1}$

$$
\left[\begin{array}{llll}
\mathbf{G}_{1}^{1} & \mathbf{G}_{1}^{e x} & \mathbf{G}_{1}^{2} & \mathbf{G}_{c 1}
\end{array}\right]\left[\begin{array}{c}
\mathbf{q}_{1}^{1} \\
\mathbf{q}_{1}^{e x} \\
\mathbf{q}_{1}^{2} \\
\mathbf{q}_{c 1}
\end{array}\right]=\left[\begin{array}{llll}
\mathbf{H}_{1}^{1} & \mathbf{H}_{1}^{e x} & \mathbf{H}_{1}^{2} & \mathbf{H}_{c 1}
\end{array}\right]\left[\begin{array}{c}
\mathbf{T}_{1}^{1} \\
\mathbf{T}_{1}^{e x} \\
\mathbf{T}_{1}^{2} \\
\mathbf{T}_{c 1}
\end{array}\right]
$$

- for sub-domain $\Omega_{2}$

$$
\left[\begin{array}{llll}
\mathbf{G}_{c 2} & \mathbf{G}_{2}^{3} & \mathbf{G}_{2}^{i n} & \mathbf{G}_{2}^{4}
\end{array}\right]\left[\begin{array}{c}
\mathbf{q}_{c 2} \\
\mathbf{q}_{2}^{3} \\
\mathbf{q}_{2}^{\text {in }} \\
\mathbf{q}_{2}^{4}
\end{array}\right]=\left[\begin{array}{llll}
\mathbf{H}_{c 2} & \mathbf{H}_{2}^{3} & \mathbf{H}_{2}^{i n} & \mathbf{H}_{2}^{4}
\end{array}\right]\left[\begin{array}{c}
\mathbf{T}_{c 2} \\
\mathbf{T}_{2}^{3} \\
\mathbf{T}_{2}^{i n} \\
\mathbf{T}_{2}^{4}
\end{array}\right]
$$

The condition (4) written in the form

$$
\left\{\begin{array}{c}
\mathbf{q}_{c 1}=-\mathbf{q}_{c 2}=\mathbf{q} \\
\mathbf{T}_{c 1}=\mathbf{T}_{c 2}=\mathbf{T}
\end{array}\right.
$$

should be introduced to the equations (19), (20). 
Next, coupling these systems of equations and taking into account the remaining boundary conditions one has

$$
\left[\begin{array}{cccccccc}
-\mathbf{H} & \alpha \mathbf{G}-\mathbf{H} & -\mathbf{H} & -\mathbf{H} & \mathbf{G} & \mathbf{0} & \mathbf{0} & \mathbf{0} \\
\mathbf{0} & \mathbf{0} & \mathbf{0} & -\mathbf{H} & -\mathbf{G} & -\mathbf{H} & \mathbf{G} & -\mathbf{H}
\end{array}\right]\left[\begin{array}{c}
\mathbf{T} \\
\mathbf{T} \\
\mathbf{T} \\
\mathbf{T} \\
\mathbf{q} \\
\mathbf{T} \\
\mathbf{q} \\
\mathbf{T}
\end{array}\right]=\left[\begin{array}{c}
\alpha \mathbf{G} T \\
\mathbf{G} T
\end{array}\right]
$$

Finally, the system of equations (22) can be written in the form

$$
\mathbf{A Z}=\mathbf{B}
$$

where $\mathbf{A}$ is the main matrix, $\mathbf{Z}$ is the unknown vector and $\mathbf{B}$ is the vector of the righthand side. The system of equations (23) allows one to find the "missing" boundary values. Knowledge of nodal boundary temperatures and heat fluxes constitutes a basis for determination of internal temperatures at the optional set of points selected from the domain considered $[2,3]$.

\section{Inverse problem}

The inverse problem considered here is based on the assumption that the temperature distribution at the boundary $\Gamma_{e x}$ is known, while the position of $\Gamma_{c}$ is unknown. This problem is solved using the gradient method [4-7].

As mentioned above, the surface $\Gamma_{c}$ is described by the NURBS curve (5). The aim of investigations is to determine the value of co-ordinate $y_{1}$ of control point $\mathbf{P}_{1}=\left(0.01, y_{1}\right)$. Let $b=y_{1}$ is the shape parameter.

The criterion which should be minimized is of the form

$$
S(b)=\frac{1}{M} \sum_{i=1}^{M}\left(T_{i}-T_{d i}\right)^{2}
$$

where $T_{d i}, T_{i}$ are the known from the measurements and calculated temperatures, respectively.

Using the necessary condition of optimum, one obtains

$$
\sum_{i=1}^{M}\left(T_{i}-T_{d i}\right) \frac{\partial T_{i}}{\partial b}=0
$$


The function $T_{i}$ is expanded into the Taylor series taking into account the first derivatives

$$
T_{i}=T_{i}^{k}+U_{i}^{k}\left(b^{k+1}-b^{k}\right)
$$

where

$$
U_{i}^{k}=\left(\frac{\partial T_{i}}{\partial b}\right)_{b=b^{k}}
$$

are the sensitivity coefficients, $k$ is the number of iteration, for $k=0 b^{0}$ is the arbitrary assumed value of parameter $b$, while for $k>0$ it results from previous iteration.

Substituting (26) into (25) one has

$$
\sum_{i=1}^{M}\left(T_{i}^{k}-T_{d i}\right) U_{i}^{k}+\left(b^{k+1}-b^{k}\right) \sum_{i=1}^{M}\left(U_{i}^{k}\right)^{2}=0
$$

hence

$$
b^{k+1}=b^{k}+\frac{\sum_{i=1}^{M}\left(T_{i}^{k}-T_{d i}\right) U_{i}^{k}}{\sum_{i=1}^{M}\left(U_{i}^{k}\right)^{2}}, \quad k=0,1,2, \ldots, K
$$

where $K$ is the number of iterations.

To determine the sensitivity coefficients (27) the following formula is used [3]

$$
U_{i}^{k}=U\left(x_{i}, y_{i}, b^{k}\right)=\frac{T\left(x_{i}, y_{i}, b^{k}+\Delta b^{k}\right)-T\left(x, y, b^{k}\right)}{\Delta b^{k}}
$$

where $\Delta b^{k}=10^{-4} b^{k}$ is a small increase in parameter $b^{k}$.

This approach requires in each iteration to solve two direct problems with the parameters $b^{k}$ and $b^{k}+\Delta b^{k}$, respectively.

\section{Results of computations}

The rectangular domain of dimensions $2 L \times L(L=0.02 \mathrm{~m})$ shown in Figure 1 has been considered. The following input data have been assumed: thermal conductivities $\lambda_{1}=2.5 \mathrm{~W} /(\mathrm{mK}), \lambda_{2}=1 \mathrm{~W} /(\mathrm{mK})$, heat transfer coefficient 
$\alpha=50 \mathrm{~W} /\left(\mathrm{m}^{2} \mathrm{~K}\right)$, ambient temperature $T_{a}=20^{\circ} \mathrm{C}$ (c.f. condition (2)), boundary temperature $T_{b}=100^{\circ} \mathrm{C}$ (c.f. condition (3)).

At first, the direct problem described in the Section 1 is solved. In Figure 2 the temperature distribution on the external surface of the domain is presented.

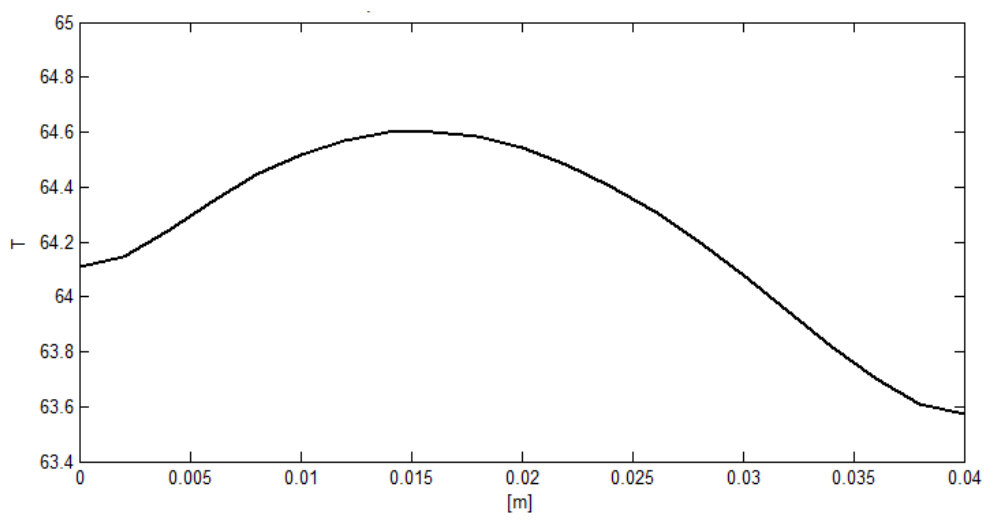

Fig. 2. Temperature distribution on the external surface

Next, the inverse problem is considered. It is assumed that the values of temperatures on the external surface (Fig. 2) resulting from the solution of direct problem are given. The inverse problem is solved under the assumption that the initial position of internal boundary is described by the NURBS curve (5) marked in Figure 3 as 'initial'.

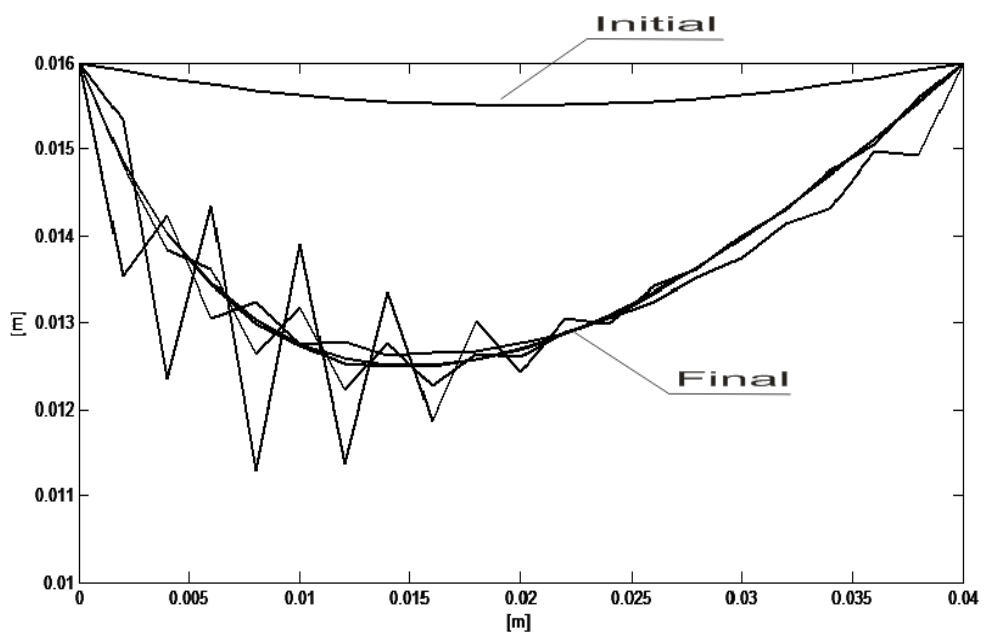

Fig. 3. Results of identification

It is visible that the iteration process is convergent and after several iterations the real position of the internal boundary is obtained. 


\section{Conclusions}

The non-homogeneous domain from two sub-domains compound has been considered. The temperature distribution has been described by the system of two Laplace equations. The inverse problem has been solved by means of BEM and the gradient method. The algorithm proposed allows one to identify the unknown position of surface between two sub-domains.

\section{References}

[1] Freus K., Freus S., Determination of temperature field in domain of complex shape using the NURBS curves and BEM, Scientific Research of the Institute of Mathematics and Computer Science 2008, 1(7), 43-48.

[2] Brebbia C.A., Domingues J., Boundary Elements, Introductory Course, CMP, McGraw-Hill Book Company, London 1992.

[3] Majchrzak E., Boundary Element Method in Heat Transfer, Publ. of the Techn. Univ. of Czest., Czestochowa 2001 (in Polish).

[4] Kurpisz K., Nowak A.J., Inverse Thermal Problems. Computational Mechanics Publications, Southampton-Boston 1995.

[5] Freus S., Freus K., Majchrzak E., Mochnacki B., Identification of internal boundary position in two-layers domain on the basis of external surface temperature distribution, CD-ROM Proceedings of the $6^{\text {th }}$ European Congress on Computational Methods in Applied Sciences and Engineering (ECCOMAS 2012) September 10-14, 2012, Vienna, Austria, Eds.: J. Eberhardsteiner, H.J. Bohm, F.G. Rammerstorfer, Publisher: Vienna University of Technology, Austria.

[6] Romero Mendez R., Jimenez-Lozano J.N., Sen M., Gonzalez F.J., Analytical solution of Burndepth determination from infrared thermographs, Mathematical Medicine and Biology 2010, (27), 21-38, doi: 1093/imammb/dqp010.

[7] Majchrzak E., Determination of burn depth on the basis of skin surface temperature - solution of inverse problem using the gradient method, $10^{\text {th }}$ World Congress on Computational Mechanics, 8-12 July 2012, Sao Paulo, Brasil, CD ROM Proceedings, 1-12. 